\title{
An MPC with disturbance forecasting for the control of the level of a tank with limited buffer capacity
}

\author{
Francesco Borghesan ${ }^{1}$, Moncef Chioua ${ }^{2}$, and Nina F. Thornhill ${ }^{1}$, Senior Member IEEE
}

\begin{abstract}
The paper deals with the behavior of an MPC for the control of a level of a tank, whose inflow is subject to persistent plantwide disturbances. It is shown that the response of an industrial MPC can be aggressive and oscillatory in such situations. The result is that the disturbance propagates further. The reason is the assumption made by the industrial MPCs regarding the future evolution of the disturbance. To improve the response in presence of plantwide disturbances, an MPC with disturbance forecasting is proposed. Such MPC is able to handle tight constraints and still reduce the movement of the outflow of the tank, therefore reducing the disturbance propagation. To compare the MPC with prediction forecasting with other two strategies used in industrial practice to handle measured disturbances, the paper uses sinusoidal disturbances and real disturbances coming from a refinery.
\end{abstract}

\section{INTRODUCTION}

In process plants, a disturbance is an undesired, transitory deviation of a process variable from its desired set point. Such disturbances can affect the quality of the product, or they can cause a malfunction of the site machinery and accelerate its wear [1]. Further, a disturbance originating from one unit can propagate to other units because of material, energy or information and control interconnections.

Control systems have a role in propagating a disturbance. A controller compensates the effect of the disturbance on the controlled variable by adjusting the manipulated variable. However, the manipulated variable can be an external disturbance for another plant unit. In this way, the plantwide disturbance continues to propagate.

The controller should manage the trade-off between rejecting the disturbance from the most critical controlled variables, and avoiding propagating the disturbance further. However, various disturbances, each characterised by its own time trend, can affect a plant over time. Therefore, there should be an optimal control action for each disturbance. For this, it would be useful for the control algorithm to be able to detect that a disturbance is occurring and to predict the future evolution of the disturbance.

The present paper implements a disturbance forecast based on the weighted version of $k$-nearest neighbour method

*Financial support is gratefully acknowledged from the Marie Skłodowska Curie Horizon 2020 EID-ITN project "PROcess NeTwork Optimization for efficient and sustainable operation of Europe's process industries taking machinery condition and process performance into account - PRONTO", Grant agreement No 675215.

${ }^{1}$ F. Borghesan and N. F. Thornhill are with the Chemical Engineering Department, Imperial College London, London, SW7 2AZ, UK (email: f.borghesane imperial.ac.uk)

${ }^{2} \mathrm{M}$. Chioua is with the ABB Corporate Research Center Germany, Ladenburg, 68526, Germany in an MPC framework. The case study is based on the benchmark model in [2] and consists of a heated tank whose inflow is affected by persistent disturbances. In this paper, the aim of the MPC with disturbance forecast is to stop the propagation of a disturbance coming from the inflow, while keeping the level within tight constraints. In particular, the paper compares the response of the MPC provided of a disturbance forecaster with other strategies used by industrial MPCs to handle measured disturbances. It will be shown how the response of the MPC can be enhanced by providing the controller with a disturbance forecast.

Section II examines the background and motivation and Section III describes the case study used for this work. Section IV shows the MPC response first to purpose made sinusoidal disturbances, and then it shows the response to disturbances based on real measured time trends from a refinery. The paper ends with a discussion and conclusions.

\section{BACKGROUND AND MOTIVATION}

Being able to predict the future evolution of a disturbance can improve the performance of a controller. In process systems, feedforward control is used routinely to compensate for measured disturbances when they are detected [3], [4], but the standard implementation of feedforward control does not include a prediction of the future evolution of the disturbance. Koerber and King [5], and Mehra et al. [6] described how previewing a disturbance improved the control action with applications in wind turbines and automotive industries. However, modelling and predicting a disturbance remains an open question. Literature provides many methods such as ARIMA models and Artificial Neural Networks [7]. The methods are classified into first principle models, statistical methods and data-driven methods. Difficulties in choosing the method arise mainly when the disturbances are caused by non-linear effects such as limit cycles, or when they arise because of random events.

Borghesan et al. [8] proposed two versions of a $k$-nearest neighbours method to forecast the future trend of persistent disturbances: a weighted and an unweighted version. The methods have been able to provide good predictions of time trends coming from a refinery.

The disturbance forecast based on the weighted version of the $k$-nearest neighbour method is implemented an MPC framework in the present paper. The MPC should stop the propagation of a plantwide disturbance by minimizing the variation of the outflow of a tank affected by a persistent disturbance of the inflow. To stop the disturbance propaga- 
tion, the outlet flow of the tank should be change only if necessary, that is only if the level is going to violate the provided constraints. Therefore, the tank works as buffer tank. However, the imposed constraints are tight. The reason is that the use of buffer tanks has decreased in the recent years [9]. Therefore, a realistic scenario is that the propagation of the disturbance should be stopped by optimally exploiting the limited buffer capacity of other process units, for example, distillation columns.

The idea of using an optimal control to control buffer tanks is not new. Campo and Morari [10] proposed the MPC as control framework to maintain the level of a buffer tank within the constraints while minimizing the change of the outflow. However, their paper considered the future values of the inlet flow equal to the present value. Since the assumption on the future evolution of the inlet flow is not precise, it could happen that the MPC does not find a feasible solution of the control action that keeps the level within its constraints. In [11] and [12], Li et al. used a stochastic MPC framework to keep the outflow of a buffer tank as constant as possible while the inflow is subject to disturbances. The inflow is defined by a mean value and a standard deviation for each instant of time. However, their work does not forecast in real time the pattern of disturbance that is occurring.

Furthermore, this paper highlights the response of an MPC to persistent plantwide disturbances. The reason is that, as Darby and Nikolaou [13] state, the performance degradation of an MPC in a process plant is often linked to the presence of an oscillating disturbance entering in the plant section controlled by the MPC. Therefore, the response of the proposed MPC with disturbance forecast is compared with other strategies used by industrial MPCs to manage measured disturbances. Such strategies are ignoring the presence of a disturbance and considering the future values of a disturbance equal to the present value [4].

\section{CASE STUDY}

\section{A. Physical model}

Fig. 1 shows the considered case study, which consists of a tank filled with water, a cold-water inflow and a water outflow. The level is the only controlled variable and the outflow valve of the tank is the only manipulated variable available to the controller.

This case study has used the dynamics of the level [2]. However, in [2], the outflow valve was modelled to be fixed at $50 \%$ of its maximal aperture. In this paper, it is modelled as a controllable valve.

The original relation of the outflow was found empirically and was (for the valve opening $\mathrm{z}$ equal to $50 \%$ and a certain level $x$ )

$$
F_{\text {out }}(z=50, x)=10^{-4}(0.1013 \sqrt{(55+x)}+0.0237)
$$

To model the effect of the valve position in the present paper, the flow has been considered as $F_{\text {out }}(z)=C_{v}(z) \sqrt{\Delta P}$. In this expression, $C_{v}(z)$ is the valve flow coefficient dependent on the valve position, $z$, and $\Delta P=P_{\text {in }}-P_{\text {out }}$ is the

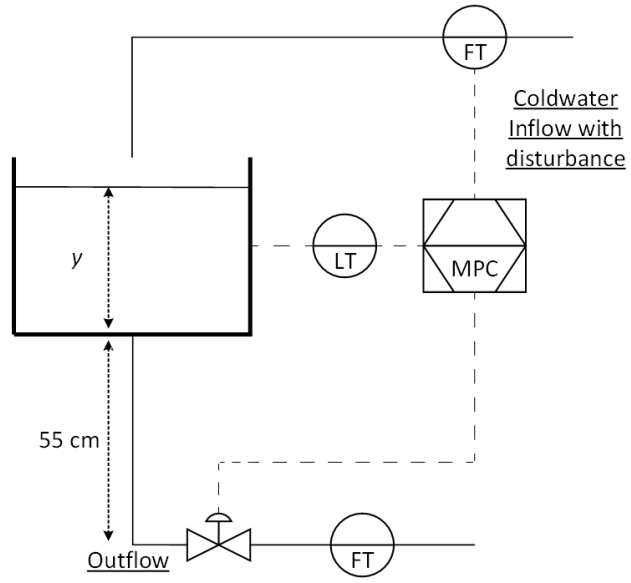

Fig. 1: Case study. The MPC receives measurements of level and of cold water disturbance and sends its output to the outflow valve.

differential pressure over the valve. The differential pressure over the valve is proportional to the height of water above the valve. Like in the original model, the valve is $55 \mathrm{~cm}$ under the bottom of the tank. Therefore $\Delta P \propto(55+x)$, where $x$ is level of the tank in $\mathrm{cm}$. Furthermore, if the characteristic of the valve is linear, then $C_{v}(z)=(a z+b) C_{v}^{M a x}$, where $C_{v}^{M a x}$ is the maximum valve flow coefficient for the valve completely open, and $a$ and $b$ are coefficients to be found. Setting $b=0$ and neglecting the bias term 0.0237 that was found in [2], the new modelled relation is:

$$
F_{\text {out }}(z, x)=\frac{0.1013(10)^{-4}}{50} z \sqrt{55+x}
$$

where $z \in[0 ; 100]$. The valve position $z$ has the following dynamic, that can be expressed in the frequency domain as

$$
z(s)=\frac{e^{-s}}{(3.8 s+1) u(s)}
$$

where $u(s)$ is the output of the controller in the range 4-20 $\mathrm{mA}$, like the measurements [2].

The inflow of the tank is considered constant in the nominal case. The disturbance $d_{C W}$ is added to the nominal value of the cold water and it considered measurable.

\section{B. Structure of the MPC}

The MPC controller has been developed using the MATLAB Model Predictive Control Toolbox. The model internal to the MPC is a linearised model of the considered nonlinear system, obtained with the Linear Analysis Tool.

The MPC controller regulates the level of the water $y$ in $\mathrm{mA}$ in the tank (whereas $x$ is the level of the water in $\mathrm{cm}$ ) by manipulating through $u$ the valve position $z$, as described in (2). The values of the disturbance $d_{C W}(N)$, sampled at the discrete instants of time $N$, are provided to the MPC as measured disturbance. Although the MPC cannot manipulate a measured disturbance, the model internal to the MPC describes the effect of the measured disturbance $d_{C W}(N)$ on the level. 
The MPC can use the data about $d_{C W}(N)$ in three possible ways

- Predicting the future values of $d_{C W}(N+h), h \geq 0$ where $N$ is the current discrete instant of time

- Considering $d_{C} W(N+h)=d_{C W}(N), \forall h \geq 0$. An MPC that uses this strategy is also called Frozen Time MPC (FTMPC) [14]

- Ignoring the presence of the disturbance. This is equivalent to setting $d_{C W}(N+h)=0, h \geq 0$

\section{Tuning of the $M P C$}

The documentation of Matlab [15] describes the QP optimization problem solved to calculate the control action of MPC. Table I lists the tuning parameters used. In particular, the weight on the controlled variable for the 'Output Reference Tracking' is set to zero because the level of the water in the tank must be free to fluctuate within the given constraints. Instead a weight has been set for the 'Move Suppression' of manipulated variable because the aim is to minimize variations of the controlled variable. The sampling time is short, because the tested disturbances have a high frequency. The prediction horizon $p=1200$ is more than the settling time of the system $(900 \mathrm{~s})$. To limit the aggressiveness of the MPC, the control horizon is short, as suggested by [16] and [17].

The resulting objective function is:

$$
\begin{aligned}
J=\sum_{i=0}^{p-1}\left\{w_{M V}\right. & {[u(N+i \mid N)} \\
& -u(N+i-1 \mid N)]\}^{2}+\rho_{\varepsilon} \varepsilon_{N}^{2}
\end{aligned}
$$

where $p$ is the prediction horizon, $w_{M V}$ is the weight for moving suppression, $u(N+i \mid N)$ is the control action (the setpoint of position of the valve outflow) planned at the discrete instant $N+i$ and calculated at the instant $N, \rho_{\varepsilon}$ is the weight for the penalization of the constraints violation, and $\varepsilon_{N}$ is the slack variable for $y$ that describes the worst violation of constraints calculated at instant $N$. The soft constraints are defined as

$$
y_{\text {Min }}-\varepsilon_{N} V_{\text {Min }}^{y} \leq y(N+i \mid N) \leq y_{\text {Max }}+\varepsilon_{N} V_{\text {Max }}^{y}
$$

$y_{M i n}$ and $y_{M a x}$ are the nominal constraints for the controlled variable (the level). $V_{\text {Min }}^{y}$ and $V_{\text {Max }}^{y}$ are adimensional tunable weights, called in the documentation ECR weights, that define how much the MPC can violate the nominal constraints. If $V_{M i n}^{y}$ and $V_{M a x}^{y}$ are set to zero, the constraints are defined in terms of hard constraints.

TABLE I: Tuning parameters of the MPC

\begin{tabular}{|c|c|}
\hline Tuning parameter & Value \\
\hline Output reference tracking & 0 \\
\hline Manipulated variable tracking & 0 \\
\hline Move suppression & 2 \\
\hline Controller sampling time & $1 \mathrm{~s}$ \\
\hline Prediction horizon & 1200 \\
\hline Control horizon & 15 \\
\hline ECR controlled variable & 1 \\
\hline
\end{tabular}

TABLE II: Constraints of the MPC

\begin{tabular}{|c|c|c|c|}
\hline Constraint & Value & Constraint & Value \\
\hline$y_{\text {Min }}$ & $12 \mathrm{~mA}$ & $y_{M a x}$ & $12.5 \mathrm{~mA}$ \\
\hline$u_{M i n}$ & $4 \mathrm{~mA}$ & $u_{M a x}$ & $20 \mathrm{~mA}$ \\
\hline$y_{M i n}^{\text {rate }}$ & $\frac{-1 \mathrm{~mA}}{\text { sample }}$ & $y_{M a x}^{\text {rate }}$ & $\frac{-1 \mathrm{~mA}}{\text { sample }}$ \\
\hline
\end{tabular}

Table II lists the constraints imposed. The constraints of the level, $y_{\text {Min }}$ and $y_{\text {Max }}$, are chosen tight. The aim is to show how also process units with little buffer capacity, like distillation columns, can reduce the disturbance propagation with adequate control. The constraints on the manipulated variable are instead relaxed. The aim is to highlight how the control action changes automatically by providing a disturbance prediction. Finally, constraints on the changing rate, $u_{\text {Min }}^{\text {rate }}$ and $u_{\text {Max }}^{\text {rate }}$ of the manipulated variable $[u(N+i \mid$ $N)-u(N+i-1 \mid N)]$ were set.

\section{Prediction forecast algorithm}

Borghesan et al. [8] proposed a weighted $k$-nearest neighbor method to predict the future evolution of a persistent disturbance.

The $k$-nearest neighbours method supposes that the current time series segment will evolve in future like a past time series segment evolved previously [18]. The algorithm uses the signal of a generic disturbance $d$ made of $N$ samples $\mathbf{d}_{M}(N)=[d(1) d(2) \ldots d(N)]$, called 'memory'. The memory size increases as more samples $d(\cdot)$ are recorded. The algorithm considers the time-series segment made of the last $m$ samples of $\mathbf{d}_{M}(N)$. This time-series segment $\mathbf{d}_{E}(N)=[d(N-m+1) d(N-m+2) \ldots d(N)]$ is called 'evolution' and it represents the current evolving disturbance pattern, while $m$ is the 'embedding dimension' [18]. The algorithm searches within the memory $\mathbf{d}_{M}(N)$ for the $k$ time series intervals of length $m$ that are most similar to $\mathbf{d}_{E}(N)$. The similarity is measured with the Euclidean distance. The $k$ time series intervals $\mathbf{d}_{j}, j=1, \ldots, k$ are the $k$-nearest neighbours and are those $k$ time series intervals within $\mathbf{d}_{M}(N)$ with smallest Euclidean distance from $\mathbf{d}_{E}(N)$ :

$$
\left\|\mathbf{d}_{E}(N)-\mathbf{d}_{j}\right\|=\sqrt{\sum_{i=1}^{m}\left(d_{E}(i)-d_{j}(i)\right)^{2}}
$$

Each nearest neighbour $\mathbf{d}_{j}$ is followed by a 'prediction contribution' $\mathbf{d}_{P j}, j=1, \ldots, k$. A 'prediction contribution' is a time series of length $h$, where $h$ is the desired prediction horizon, that constitutes the basis for building the prediction vector. The prediction contributions contribute to the prediction proportionally to distance of their respective nearest neighbour from the evolution. A weight $w_{j}$ for each nearest neighbour is calculated. The weight $w_{j}=1$ if the nearest neighbour $\mathbf{d}_{j}$ has the smallest distance with $\mathbf{d}_{E}(N)$. Instead, $w_{j}=0$ if the nearest neighbour $\mathbf{d}_{j}$ is the most distant from $\mathbf{d}_{E}(N)$.

$$
w_{j}=\frac{\max _{\ell=1 \ldots k}\left(\left\|\mathbf{d}_{E}(N)-\mathbf{d}_{\ell}\right\|\right)-\left\|\mathbf{d}_{E}(N)-\mathbf{d}_{j}\right\|}{\max _{\ell=1 \ldots k}\left(\left\|\mathbf{d}_{E}(N)-\mathbf{d}_{\ell}\right\|\right)-\min _{\ell=1 \ldots k}\left(\left\|\mathbf{d}_{E}(N)-\mathbf{d}_{\ell}\right\|\right)}
$$




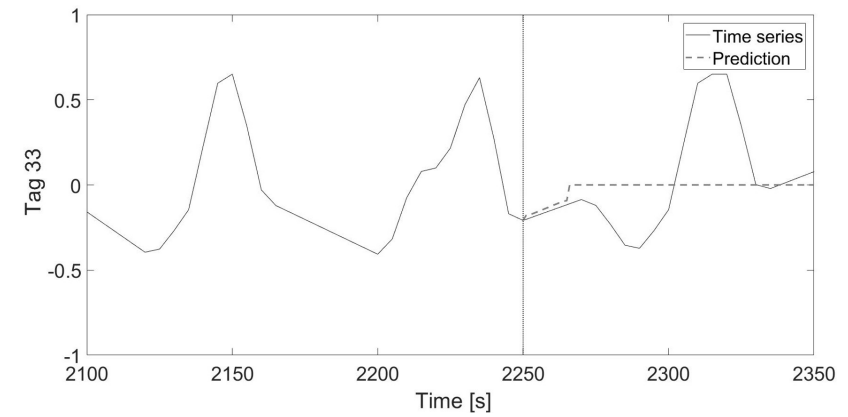

(a) Tag 33

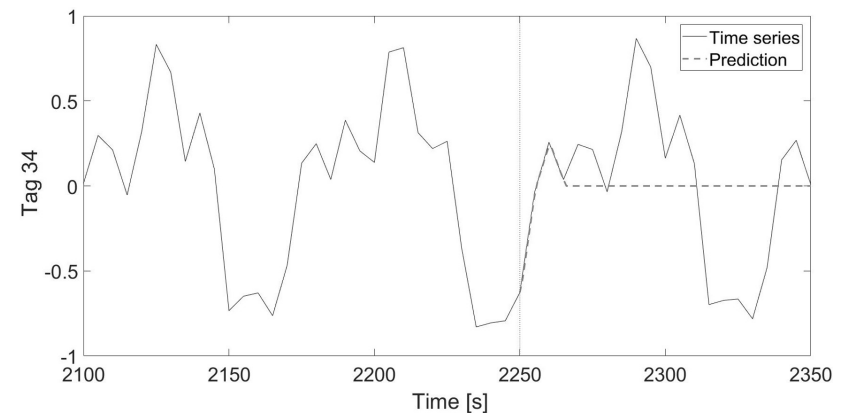

(b) Tag 34

Fig. 2: Time trend used and example of the prediction

where $j=1, \ldots, k$. If $k=1$, then $w_{1}=1$.

The prediction $\hat{\mathbf{d}}(N, h)=[\hat{d}(N+1) \ldots \hat{d}(N+h)]$ is obtained as a weighted average of the prediction contributions, where:

$$
\hat{d}(N+i)=\frac{1}{\sum_{j=1}^{k} w_{j}} \sum_{j=1}^{k} w_{j} d_{P j}(i)
$$

for $i=1, \ldots, h$.

The parameters $m$ and $k$ are the important parameters for the quality of the prediction quality. Good results were found setting $m=\left\lfloor\frac{1}{f_{0}}\right\rfloor$, where $f_{0}$ is the main frequency of the disturbance, and in setting $k=\left\lfloor\frac{N}{m}\right\rfloor$, where $\lfloor\cdot\rfloor$ is the floor function. The frequency of a persistent disturbance is constant and therefore $m$ is constant, while $k$ is updated as the size of the memory is increased.

\section{E. Implementing the disturbance forecast in the MPC frame- work}

The former section has described how a prediction of $h$ steps ahead of a persistent disturbance can be obtained. Such information must be provided to the MPC controller. The MPCToolbox of Matlab allows the MPC controller to receive a disturbance preview of the measured disturbances of the controlled system. This feature has been used to provide the MPC with the prediction of the disturbance.

$\widehat{\mathbf{d}}_{M P C}(N)$ is the vector provided to the MPC controller. Its length is $p+1$ and it is the concatenation of three parts. The first part is the measurement of the current disturbance value $d_{C W}(N)$. The second part is the prediction vector $\widehat{\mathbf{d}}_{C W}(N, h)$ with the $h$ predicted values of the disturbance. The third part is a vector $\mathbf{0}^{p-h}$ composed of $p-h$ zeros that fills the remaining elements of $\hat{\mathbf{d}}_{M P C}(N)$. Therefore, $\widehat{\mathbf{d}}_{M P C}(N)$ can be written as

$$
\begin{aligned}
\hat{\mathbf{d}}_{M P C}(N) & =\left[\begin{array}{lll}
d_{C W}(N) & \widehat{\mathbf{d}}_{C W}(N, h) & \mathbf{0}^{p-h}
\end{array}\right] \\
& =\left[\begin{array}{lll}
\widehat{d}_{M P C}(N) & \widehat{d}_{M P C}(N+1) \ldots \widehat{d}_{M P C}(N+p)
\end{array}\right]
\end{aligned}
$$

The reason for filling $\hat{\mathbf{d}}_{M P C}(N)$ with zeros is the following. Defining $r$ the length of $\hat{\mathbf{d}}_{M P C}(N)$, if $r<p+1$ the controller of MPCToolbox fills automatically the missing elements of the vectors. Precisely, it supposes that
$\hat{d}_{M P C}(N)=\hat{d}_{M P C}(N+h), i=h, \ldots, p-1$. However, if $\hat{d}_{M P C}(N+h) \neq 0$ this would imply that there is a change of the load for the level of the tank and that the outflow should be permanently changed. Instead, since persistent disturbances are considered, which do not imply necessarily a permanent change of the load, the vector $\hat{\mathbf{d}}_{M P C}(N)$ has a length of $p+1$ and $\hat{d}_{M P C}(N+i)=0, i=h, \ldots, p-1$.

Finally, it has been found during the tests that the length of disturbance forecasting should equal the control horizon, $h=$ $c$ so that the controller can counteract the known disturbance.

\section{F. Testing procedure}

To compare the different strategies, first purpose made sinusoidal time-trends and then time trends from an industrial data set were used. The sinusoidal disturbances were used to analyze the response of an MPC to a simple persistent disturbance. The industrial time trends were instead used to test the prediction algorithm proposed in conjunction with the MPC.

Using real time trend, the result is an hybrid simulation, in which two time series (Tag 33 and 34) from the SE Asia data set in [19] were used to feed the disturbance $d_{C W}$ of the CSTH model. Fig. 2 shows such time trends with an example of the prediction at instant $N=2250$. The signals have been mean-centred, scaled to their maximum absolute value and doubled in value.

$$
\tilde{\mathbf{d}}_{C W}=2 \frac{\overline{\mathbf{d}}_{C W}}{\max \left|\overline{\mathbf{d}}_{C W}\right|}
$$

where $\overline{\mathbf{d}}_{C W}$ is the mean-centered vector of $\mathbf{d}_{C W}(N)$. The time trends were made of 512 samples and it has been arbitrarily assumed that 1 sample $=5 \mathrm{~s}$. As Section III$\mathrm{D}$ described, the prediction algorithm needs a 'memory' where it can search for the nearest neighbours of the present evolution. Therefore, the initial size of the memory was made of 256 samples over 512 .

\section{RESULTS}

The result section compares the three approaches described in Section III-B to handle the measured disturbance. The aim is to highlight how the disturbance prediction can 

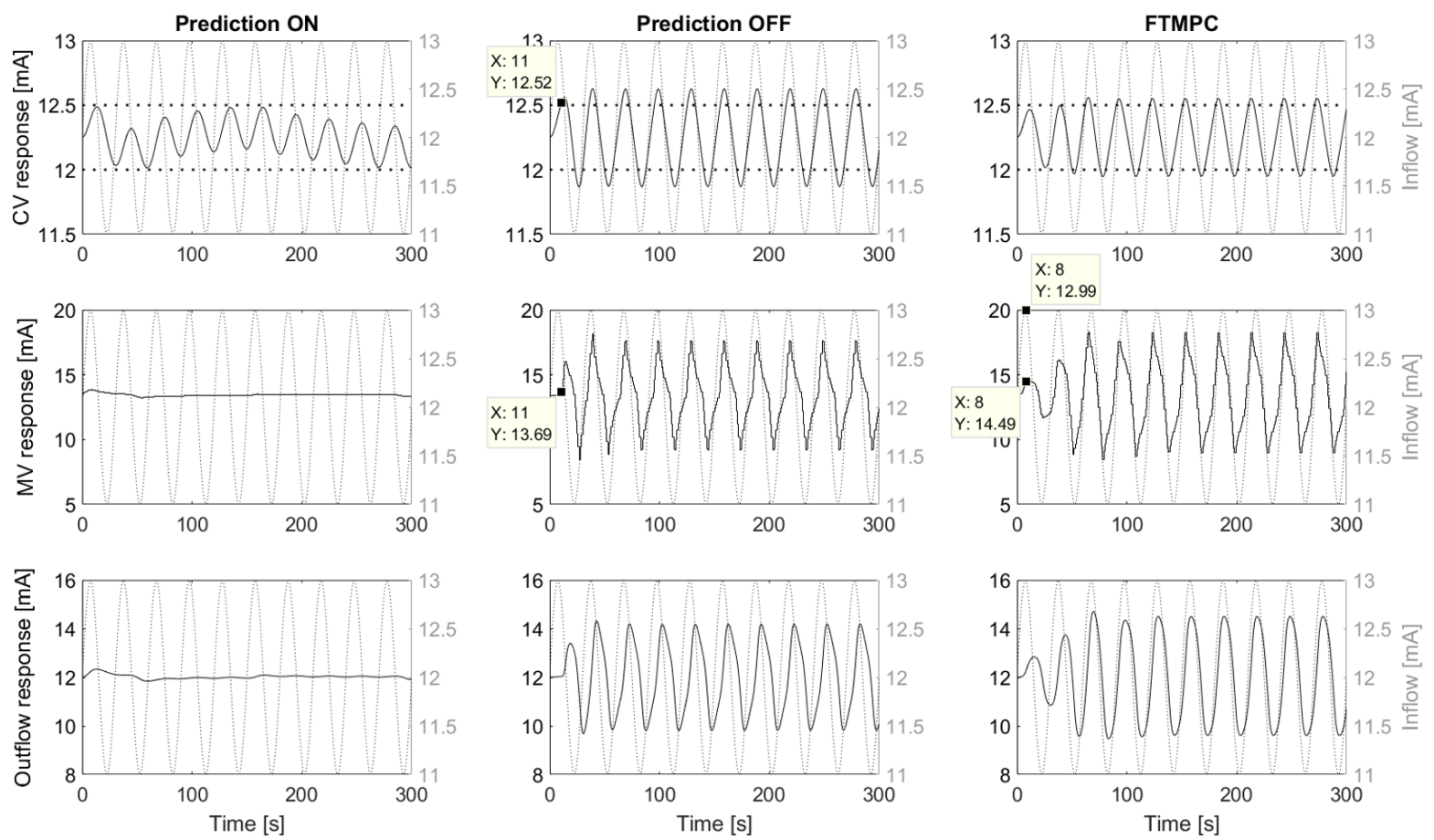

Fig. 3: Results of the responses of the level (CV), of the setpoint of the valve position (MV), and of the outflow when the frequency of the sinusoidal disturbance is $1 / 30 \mathrm{~Hz}$

(The inflow is dashed)
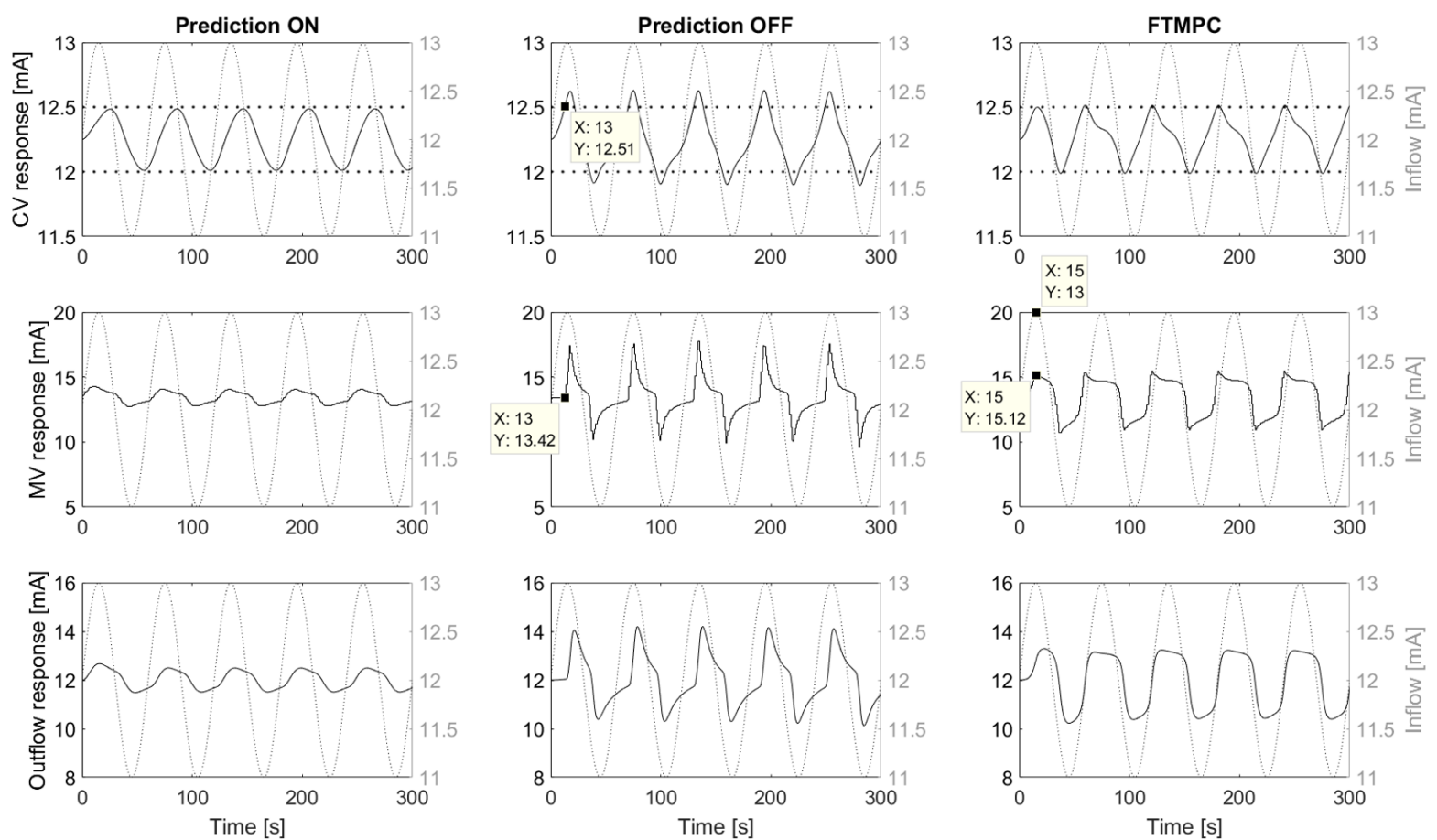

Fig. 4: Results of the responses of the level (CV), of the setpoint of the valve position (MV), and of the outflow when the frequency of the sinusoidal disturbance is $1 / 60 \mathrm{~Hz}$

(The inflow is dashed)

improve the MPC performance. Indicators of the performance are the ability of the controller to keep the level within the constraints, and to attenuate the disturbance propagation. The results section considers two experiments. In the first 

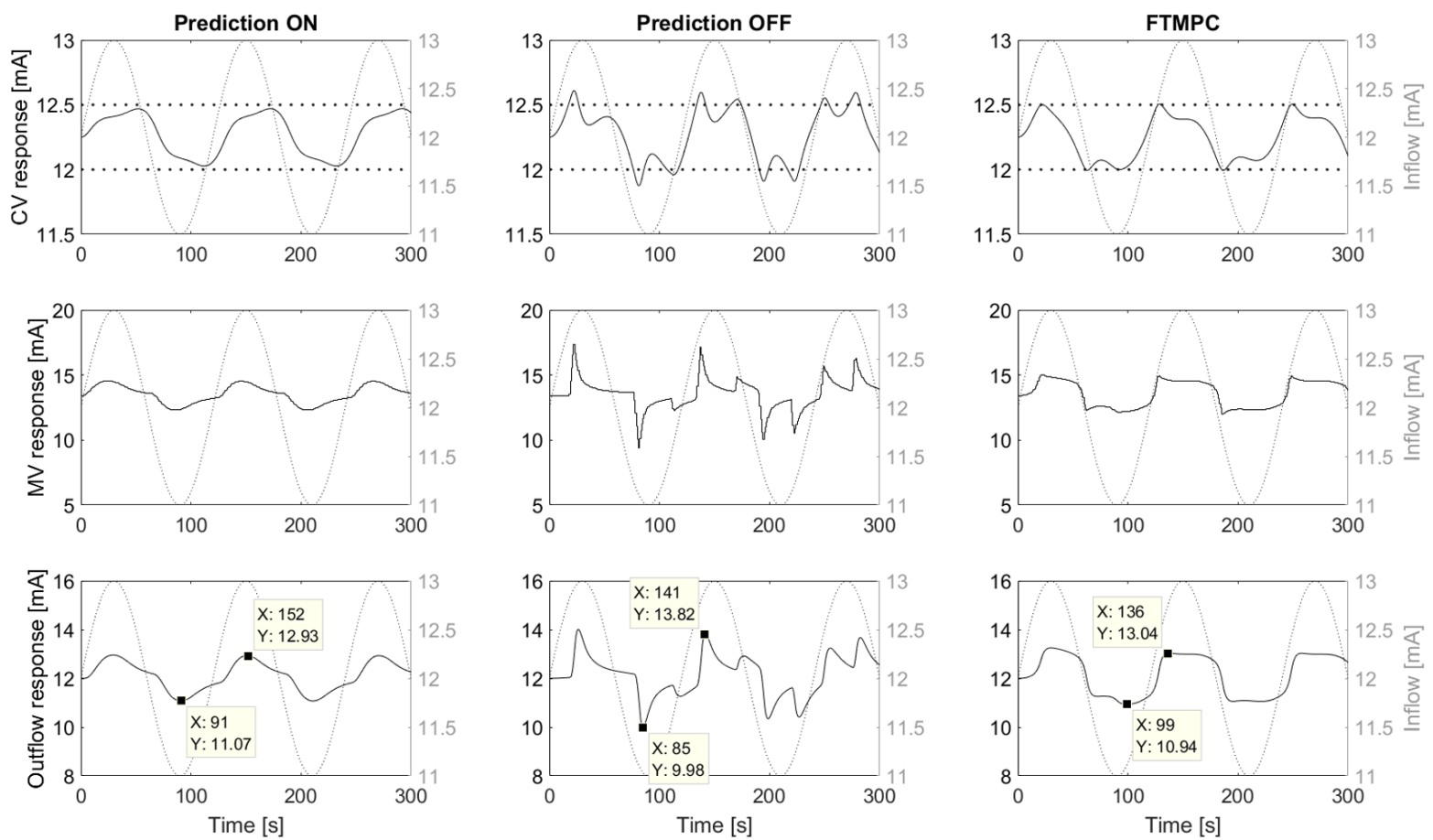

Fig. 5: Results of the responses of the level (CV), of the setpoint of the valve position (MV), and of the outflow when the frequency of the sinusoidal disturbance is $1 / 120 \mathrm{~Hz}$

(The inflow is dashed)

part, sinusoidal disturbances $d_{C W}=\sin (\omega t)$ enter in the system. The assumption is that the controller knows exactly perfectly the future evolution of the disturbance for $h=c$ steps, as Section III-E described. The scope of this test is to understand the behavior of the MPC with a very regular signal under different frequencies.

In the second part of the results section, the disturbances that enters in the system are real data coming from a SE refinery. These disturbance signals are those that have been used to test the weighted $k$-nearest neighbors method in [8].

\section{A. Sinusoidal disturbances}

Fig. 3, Fig. 4 and Fig. 5 shows the trend over time of the level, of the manipulated variable, of the outflow and, dashed, of the inflow (the disturbance) using the three MPC strategies mentioned in Section III.B for various sinusoidal disturbances $d_{C W}$. The disturbance is $d_{C W}=\sin (\omega t)$, where $\omega \in \frac{2 \pi}{30} ; \frac{2 \pi}{60} ; \frac{2 \pi}{120}$.

When the frequency of the inflow disturbance decreases, the responses of the MPCs using the traditional strategies to handle disturbances improve. Furthermore, the responses are more similar to the response of the MPC with exact information regarding the future evolution of the disturbance. However, for $\frac{2 \pi}{120}$, it can be noted that all the controllers have introduced nonlinearities in the plantwide disturbance, but the nonlinearities are smaller, when the prediction is used. Indeed, the outflow of the tank is more similar to a sinusoid (like the inflow) when the MPC knows the future evolution of disturbance, then in the cases of MPC with the 'Prediction
OFF' or in the case of FTMPC.

The explanation of the above results is the following. Since the controlled variable does not have to follow a reference value, the MPC minimizes its objective function in Eq. (3) by keeping the control action constant as long as possible in absence of constraint violations. The MPC intervenes when it detects that the constraints have been violated or are going to be violated during the prediction horizon.

The MPC whose internal model ignores the presence of the inflow disturbance, reacts when the constraints are violated. In the first instants of the simulations such MPC does not move the manipulated variable, as Fig. 3 and Fig. 4 show. As soon as the constraints are violated, the controller reacts by moving fast the manipulated variable in order to bring the level within its constraints as fast as possible. Indeed, as Eq. (3) shows, the violation of the constraints is strongly penalized, more than a change of the control action.

The result are characteristic peaks that are visible in the Fig. 4 and Fig. 5 in the rows denominated 'MV response' and 'Outflow response'. The rise per sample of manipulated variable is $\frac{1 \mathrm{~mA}}{\text { sample }}$, like the imposed constraints on the changing rate of manipulated variable. If these constraints are strengthened, the shape would look like a succession of ramps.

Once the level is again within the constraints, the controller changes only slightly the valve position. The choice is not appropriate because the disturbance oscillating at high frequency is bringing the level in the opposite direction. This 

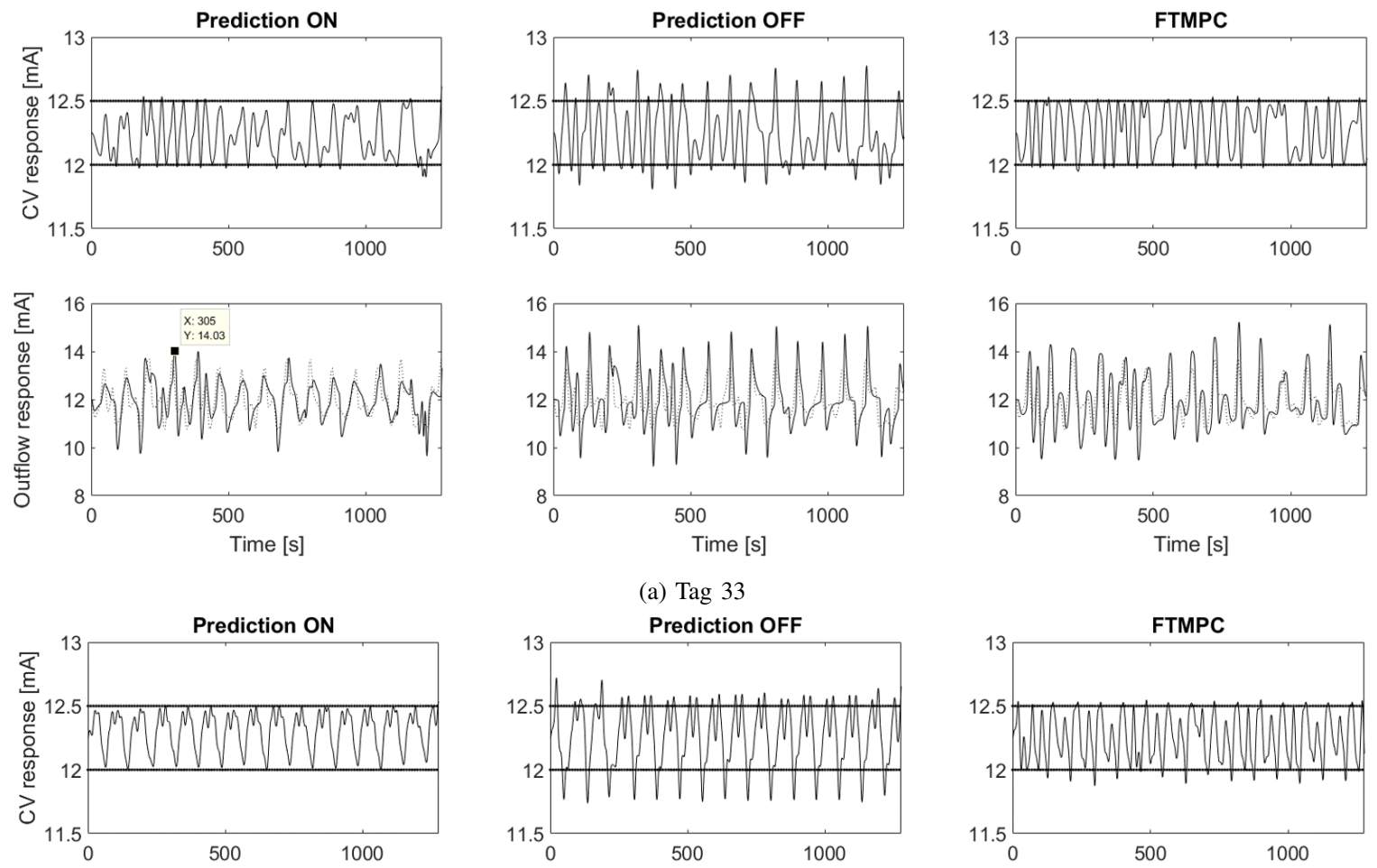

(a) Tag 33
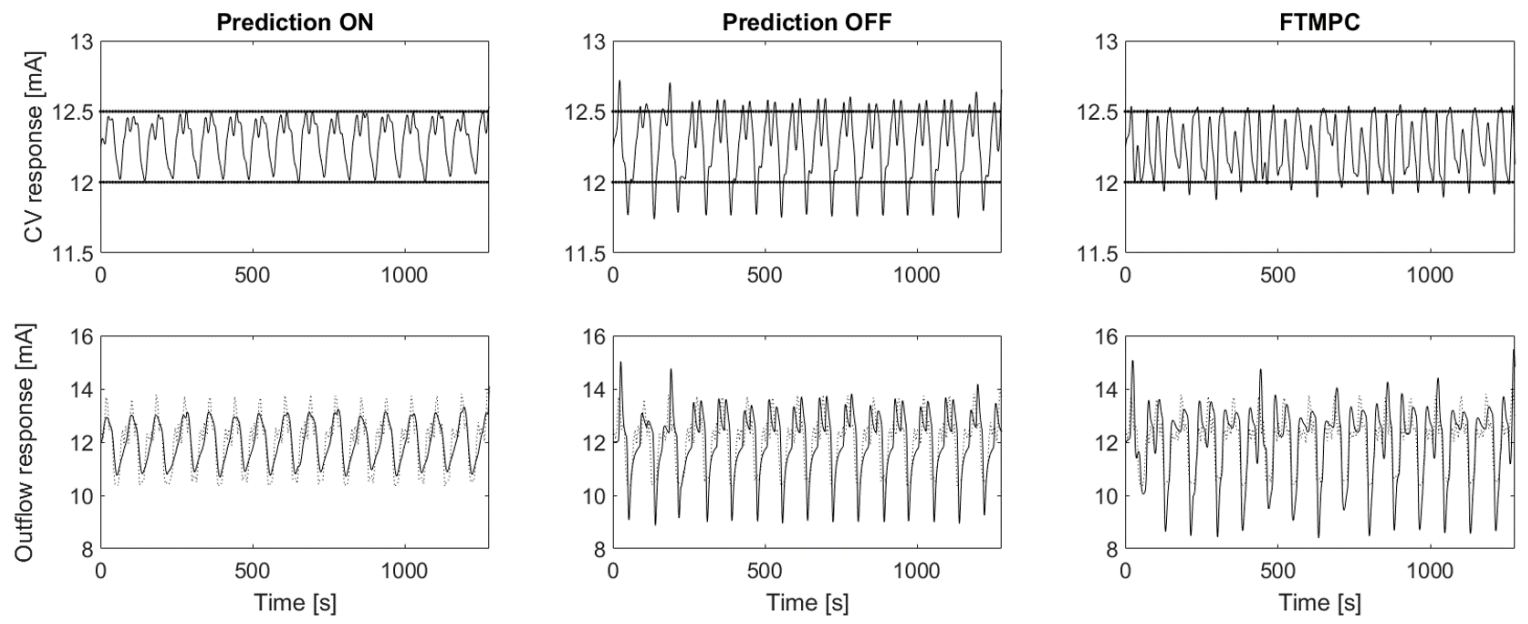

(b) Tag 34

Fig. 6: Results of the response of the level (CV) and of the outflow using real disturbances from a SE refinery (the inflow of the tank is dashed)

violates the opposite constraint. The controller intervenes aggressively moving the manipulated variable in the opposite direction. The result is a limit cycle. When the frequency of the disturbance is lower, the control action of the MPC is less aggressive because there is less interaction between the effect of the inflow and of the outflow.

The FTMPC reacts instead when it calculates that the constraints are violated in the future. For the FTMPC the variation of the inflow at each instant of time is a step variation. The effect of a step of the disturbance on the level is far more significant than the effect of a higher frequency disturbance. The reason is that the relation between inflow and level is described by a first-order transfer function that works as a low pass filter.

Under this (wrong) assumption, the FTMPC might predict that the constraints are violated in the future if the position of the outflow valve does not change. In such case, the controller must change the value of the valve outflow so that it fulfils the constraints. Fig. 3 and Fig. 4 show that the first peak of the manipulated variable coincides with the first peak of the inflow.

Thereafter a limit cycle starts. Indeed, at high frequencies, the variations of the inflow between one instant of time and the following are higher than at lower frequencies. The corresponding control action involves large movements of the manipulated variable which are too aggressive for the limited effects that a high frequency disturbance can have. The result is an aggressive control action that causes a limit cycle and violations of the constraints. If the frequency of the disturbance is slower, the control action of such MPC, designed for disturbance whose frequency is $f=0$, is more reasonable. This improves the performance of the system.

Using the information of future evolution of the disturbance for the duration of the control horizon, the MPC applies changes to the manipulated variable which are more adequate. Fig. 3 shows for example that such MPC does some small corrections to the manipulated variable before leaving it constant over time. The other two MPCs instead overreact to a constraint violation or to a future possible constraint violation, entering in a limit cycle as explained. 
TABLE III: Minimal and maximal value of the outflow for the tested inflow disturbances

\begin{tabular}{|l|r|r|r|}
\hline \multicolumn{4}{|c|}{ Tag 33 } \\
\hline & Pred. ON & Pred. OFF & FTMPC \\
\hline Max outflow & $14.04 \mathrm{~mA}$ & $15.08 \mathrm{~mA}$ & $15.18 \mathrm{~mA}$ \\
\hline Min outflow & $9.68 \mathrm{~mA}$ & $9.26 \mathrm{~mA}$ & $9.47 \mathrm{~mA}$ \\
\hline \multicolumn{4}{|c|}{ Tag 34 } \\
\hline & Pred. ON & Pred. OFF & FTMPC \\
\hline Max outflow & $13.32 \mathrm{~mA}$ & $15.03 \mathrm{~mA}$ & $15.07 \mathrm{~mA}$ \\
\hline Min outflow & $10.71 \mathrm{~mA}$ & $8.87 \mathrm{~mA}$ & $8.39 \mathrm{~mA}$ \\
\hline
\end{tabular}

\section{B. Real disturbances}

The Fig. 6a and Fig. 6b show the results of applying real time-trends on the considered case study using the proposed prediction algorithm. The MPC with prediction forecasting reduces the constraints violations for both Tag 33 and Tag 34 from [19]. Especially the MPC without any information regarding the disturbance value, violates the given constraints with both disturbances. The FTMPC violates less the constraints but its control action is aggressive.

The MPC with disturbance forecasting is instead able to fulfill both requirements of keeping the level within the given constraints and reducing the variations of the outflow. It is particularly successful in case of Tag 34. The reason might be that the prediction of the Tag 34 is more accurate because the time trend is more regular. Table III list the highest and lowest peak values of the measured outflow using the considered strategies to handle the disturbance.

\section{CONCLusions}

Plantwide disturbances can propagate because of the response of controllers to the propagating disturbance. Since disturbances characterized by different time trends can affect a plant over time, an optimal control action would be needed for each disturbance to handle the trade-off between keeping the controlled variable with desired values and avoiding further propagation of the disturbance. This paper proposes an MPC that works in conjunction with a disturbance predictor presented in [8]. The case study derives from [2], and it consists of a tank filled with water, whose inflow is affected by a disturbance. The aim of the controller is to reduce the variations of the manipulated outflow, keeping the level of the water within two constraints. The considered disturbance are purpose made sinusoidal disturbances and two time series from the SE Asia data set in [19]. A comparison is made with two other industrial strategies used to handle disturbances. Such strategies are ignoring the presence of a disturbance and considering the future values of the disturbance equal to present value.

The present paper highlights how the response of an industrial MPC to a persistent plantwide disturbance characterized by a high frequency can be oscillatory and have significant variations over time. This might be one of the reason why, as Darby and Nikolaou [13] state, the performance degradation of an MPC in a process plant is often linked to the presence of an oscillating disturbance entering in the plant section controlled by the MPC. Further this paper shows that together with a disturbance prediction, not only the inadequate response of the MPC is mitigated, but also that an MPC can even stop the propagation of a plantwide disturbance.

\section{REFERENCES}

[1] T. Yuan and S. J. Qin, "Root cause diagnosis of plant-wide oscillations using granger causality," Journal of Process Control, vol. 24, no. 2, pp. $450-459,2014$.

[2] N. F. Thornhill, S. C. Patwardhan, and S. L. Shah, "A continuous stirred tank heater simulation model with applications," Journal of Process Control, vol. 18, no. 3-4, pp. 347-360, 2008.

[3] B. G. Liptak, "Feedback and Feedforward Control," 2005.

[4] E. Camacho and C. Bordons, Model Predicitve Control. Springer Verlag, 2008.

[5] A. Koerber and R. King, "Combined feedback-feedforward control of wind turbines using state-constrained model predictive control," IEEE Transactions on Control Systems Technology, vol. 21, no. 4, pp. 11171128, 2013.

[6] R. Mehra, J. Amin, K. Hedrick, C. Osorio, and S. Gopalasamy, "Active suspension using preview information and model predictive control," Proceedings of the 1997 IEEE International Conference on Control Applications, pp. 860-865, 1997.

[7] J. G. De Gooijer and R. J. Hyndman, "25 Years of time series forecasting," International Journal of Forecasting, vol. 22, no. 3, pp. 443-473, 2006.

[8] F. Borghesan, M. Chioua, and N. F. Thornhill, "Forecast of persistent disturbances using k -nearest neighbour methods," in PSE 2018, San Diego, 2018.

[9] W. L. Luyben, M. L. Luyben, and B. D. Tyreus, Plantwide process control. McGraw-Hill Professional Publishing, 1998.

[10] P. J. Campo and M. Morari, "Model predictive optimal averaging level control," AIChE Journal, vol. 35, no. 4, pp. 579-591, 1989.

[11] P. Li, M. Wendt, H. Arellano-Garcia, and G. Wozny, "Optimal operation of distillation processes under uncertain inflows accumulated in a feed tank," AIChE Journal, vol. 48, no. 6, pp. 1198-1211, 2002.

[12] P. Li, H. Arellano-Garcia, and G. Wozny, "Chance constrained programming approach to process optimization under uncertainty," Computers \& Chemical Engineering, vol. 32, no. 1-2, pp. 25-45, 2008.

[13] M. L. Darby and M. Nikolaou, "MPC: Current practice and challenges," Control Engineering Practice, vol. 20, no. 4, pp. 328-342, 2012.

[14] S. D. Cairano, D. Bernardini, A. Bemporad, and I. V. Kolmanovsky, "Stochastic MPC with learning for driver-predictive vehicle control and its application to HEV energy management," IEEE Transactions on Control Systems Technology, vol. 22, no. 3, pp. 1018-1031, 2014.

[15] Mathworks, "Optimization Problem - Documentation Matlab 2017b," 2017. [Online]. Available: https://de.mathworks.com/help/mpc/ug/optimization-problem.html

[16] - "Choose Sample Time and Horizons." [Online]. Available: https://de.mathworks.com/help/mpc/ug/choosing-sampletime-and-horizons.html, urldate $=2018-02-04$

[17] D. E. Seborg, T. F. Edgar, and D. A. Mellichamp, Process Dynamics and Control, second edi ed. John Wiley \& Sons, 2004.

[18] H. Kantz and T. Schreiber, Nonlinear Time Series Analysis, 2nd ed. Cambridge: Cambridge University Press, 2004.

[19] N. F. Thornhill, "Finding the source of nonlinearity in a process with plant-wide oscillation," IEEE Transactions on Control Systems Technology, vol. 13, no. 3, pp. 434-443, 2005. 